\title{
Variation in thyroid volumes due to differences in the measured length or area of the cross-sectional plane: A validation study of the ellipsoid approximation method using CT images.
}

\section{Naotoshi Fujita}

Nagoya University Graduate Shool of Medicine

Katsuhiko Kato ( $\sim$ katokt@med.nagoya-u.ac.jp )

Nagoya University Graduate School of Medicine https://orcid.org/0000-0002-2083-9319

Shinji Abe

Nagoya University Hospital

Shinji Naganawa

Nagoya University Graduate School of Medicine

Original research

Keywords: CT, Ellipsoid approximation, Graves' disease, Internal radioiodine therapy, Thyroid, Volumetry

Posted Date: March 26th, 2020

DOl: https://doi.org/10.21203/rs.3.rs-19192/v1

License: (c) (i) This work is licensed under a Creative Commons Attribution 4.0 International License.

Read Full License

Version of Record: A version of this preprint was published at Journal of Applied Clinical Medical Physics on March 29th, 2021. See the published version at https://doi.org/10.1002/acm2.13125. 
2 Variation in thyroid volumes due to differences in the measured length or area of the cross-

3 sectional plane: A validation study of the ellipsoid approximation method using CT images.

4

$5 \quad$ Article type

6 Original Research Article

7

$8 \quad$ All authors' full name

9 Naotoshi Fujita ${ }^{1,2}$, Katsuhiko Kato ${ }^{2, *}$, Shinji Abe ${ }^{1}$ and Shinji Naganawa ${ }^{3}$

11 Affiliations and addresses

$12{ }^{1}$ Department of Radiological Technology, Nagoya University Hospital, 65 Tsurumai-cho, 13 Showa-ku, Nagoya, 466-8560, Japan

$14{ }^{2}$ Department of Radiological and Medical Laboratory Sciences, Nagoya University Graduate 15 School of Medicine, 1-1-20 Daiko-Minami, Higashi-ku, Nagoya, 461-8673, Japan

$16{ }^{3}$ Department of Radiology, Nagoya University Graduate School of Medicine, 65 Tsurumai17 cho, Showa-ku, Nagoya, 466-8550, Japan

\section{*Corresponding author}

20 Katsuhiko Kato

Department of Radiological and Medical Laboratory Sciences, Nagoya University Graduate

22 School of Medicine, 1-1-20 Daiko-Minami, Higashi-ku, Nagoya, 461-8673, Japan

TEL: +81-52-719-1590

24

E-mail: katokt@med.nagoya-u.ac.jp 
Abstract

\section{Background}

31 This study examined the variation in the thyroid volume determined by the ellipsoid approximation method due to differences in the measured length or area of the cross-sectional plane of CT images.

\section{Methods}

Forty-five patients with Graves' disease were included in this retrospective study. We designated the three-dimensional thyroid volumes extracted manually $\left(V_{\mathrm{CT}}\right)$ as the reference data and calculated five approximate volumes for comparison: (1) the mean volume of 8100 different thyroid volumes depending on the diameter of the cross-sectional plane at the midpoint of the major axis, $\left(V_{\text {ellipsoid,mean }}\right)$; (2) the volume using the maximum diameter and its orthogonal diameter, ( $\left.V_{\text {ellipsoid,maxlength }}\right)$; (3) the maximum ( $\left.V_{\text {ellipsoid,maxvolume }}\right)$ and (4) minimum ( $\left.V_{\text {ellipsoid,minvolume }}\right)$ of the 8100 thyroid volumes; and (5) the volume determined with an equivalent circle diameter, $\left(V_{\text {ellipsoid,Heywood }}\right)$.

\section{$43 \quad$ Results} utilizing three-dimensional thyroid volumetry if measurement accuracy is required.

\section{Keywords}


Internal radioiodine therapy (iodine-131) is a treatment for Graves' disease [1]. In radioiodine therapy for Graves' disease, the administered radioactivity is often determined based on the patient's thyroid volume [2-4]. Specifically, patients with Graves' disease who have large thyroid volumes will require a higher dose to ensure a therapeutic effect [3-4]. Therefore, accurate thyroid volumetry before radioiodine therapy leads to the accurate determination of the administered radioactivity, thereby ensuring the therapeutic effect. Thyroid volumetry involves analyses of images obtained from: (1) scintigraphy [5-6], (2) ultrasonography [7], and (3) computed tomography (CT) [8-10]. Utilizing these images, the thyroid is approximated as a complex of ellipsoids, known as the ellipsoid approximation method, and it has been in popular use owing to its inherent simplicity. In the ellipsoid approximation method, it is customary to use the maximum diameter of the cross-sectional plane (transverse plane of the body), although there is no clear rule in either past papers or guidelines. Schlögl et al. compared the accuracy of the thyroid volume determined by the ellipsoid approximation method and the three-dimensional segmentation of ultrasound images [11]. In their study, the ellipsoid volumes were determined by measuring the maximum transversal, horizontal, and longitudinal diameters. They reported that the approximate volume was $11 \%$ larger than the actual volume with a standard deviation of $26 \%$. However, they did not report on the reason for using the maximum diameter, the method used to measure each diameter, and its variation. We believe the differences in the measured length or area of the cross-sectional plane particularly affect the ellipsoid and thus the approximated thyroid volume. Furthermore, the accuracy of the measurements depends on the subjectivity and skill of the measurers (i.e., physicians, radiological technologists, etc.). The results of three-dimensional thyroid volumetry from CT images were reported to be in good agreement with the actual thyroid volume by Lee et al. [10]. They targeted patients who underwent contrast-enhanced CT examination, and the thyroid regions were extracted from the CT images using a three-dimensional visualization software. However, contrast-enhanced CT scans will delay radioiodine therapy for weeks or months because the contrast media contains iodine [3-4]. Therefore, direct application of their 
85 approach is difficult for patients with Graves' disease before radioiodine therapy.

Regardless of the modality employed, it is significant to validate an accurate and

87 simple method for measuring the thyroid volume. We need to investigate how the thyroid

88 volume changes with various diameter combinations, including the combination of the

89 maximum and orthogonal diameter of the cross-sectional plane. This study aimed to calculate

90 the variation in the thyroid volume determined by the ellipsoid approximation method due to

91 differences in the measured length or area of the cross-sectional plane of CT images. To the

92 best of our knowledge, similar studies have not been reported.

93

94

95

96

97

98

99

100

101

102

103

104

105

106

107

108

109

110

111

112 


\section{$114 \quad$ Patients}

115 Forty-five patients (7 males, 38 females, average patient age of $50.8 \pm 16$ years) with Graves' 116 disease who underwent radioiodine therapy as outpatients from December 2014 to April 2018 117 were included in this retrospective study, approved by the Ethical Review Committee of 118 Nagoya University Hospital (authorization no. 2018-0179). Because five of this cohort underwent radioiodine therapy twice during this period, our analysis eventually included 50 cases. In our hospital, we determined the administered radioactivity of radioiodine for Graves' disease according to Marinelli's formula [2]. Using Marinelli's formula (Eq. 1), the administered radioactivity, $A$, was calculated using the thyroid volume, amongst other parameters. For this reason, patients underwent non-enhanced neck CT examinations (Aquilion 64 or Aquilion PRIME SP; Canon Medical Systems, Otawara, Japan) for the determination of thyroid volumes. In this study, we performed retrospective analyses using these neck CT images.

The Nuclear Medicine physician determines the administered radioactivity by adjusting the absorbed dose in Equation 1 according to the clinical symptoms of each patient.

$$
A[\mathrm{MBq}]=C \times \frac{D \times V}{U \times T_{\mathrm{eff}}}
$$

where $A$ is the administered radioactivity (MBq), $D$ is the thyroid absorbed dose (Gy), $V$ is the thyroid volume $(\mathrm{mL}), U$ is the radioiodine uptake fraction at 24 hours after administration, $T_{\text {eff }}$ is the effective iodine-131 half-life (d), and $C$ is the unit conversion coefficient (MBq d Gy ${ }^{-1}$ $\mathrm{g}^{-1}$ ). In our hospital, we substitute 0.185 for $C$. Hereafter, the density of the thyroid is assumed to be $1 \mathrm{~g} / \mathrm{mL}$.

\section{Three-dimensional thyroid volumetry using CT images}

139 According to Shu et al. [9] and Lee et al. [10], the results of three-dimensional thyroid volumetry using CT images were in good agreement with the actual thyroid volume. Schlögl 
et al. [11] also reported similar results using ultrasound images. Therefore, we designated the three-dimensional thyroid volumes extracted manually as the reference data $\left(V_{\mathrm{CT}}\right)$ in this study.

143 First, the thyroid region of interest (ROI) in the CT images were manually extracted from the

144 CT images with a slice thickness of $5 \mathrm{~mm}$. The thyroid region was extracted while excluding 145 the surrounding blood vessels or muscles. Then, the sum of the voxels in each slice was calculated as the thyroid volume.

\section{Thyroid volumetry using ellipsoid approximation}

The ellipsoid approximation method for thyroid volumetry was described by Malago et al. [7], Lee et al. [10], and Schlögl et al. [11]. The thyroid volume of each lobe was approximated by an ellipsoid using Equation 2; then, the sum of each lobe was taken as the thyroid volume

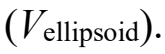

$$
V_{\text {ellipsoid }}\left[\mathrm{cm}^{3}\right]=\left(\frac{\pi}{6} \times a_{L} \times b_{L} \times c_{L}\right)_{\text {Leftlobe }}+\left(\frac{\pi}{6} \times a_{R} \times b_{R} \times c_{R}\right)_{\text {Rightlobe }}
$$

where $a(\mathrm{~cm})$ is an arbitrary diameter and $b(\mathrm{~cm})$ is its orthogonal diameter. Each line passes through the centroid of the thyroid ROI on the cross-sectional plane when the thyroid is approximated by an ellipsoid. $c(\mathrm{~cm})$ corresponds to the length of the major axis of the ellipsoid. The subscripts $L$ and $R$ indicate left and right, respectively. In this study, the isthmus was not considered in the volume calculation.

The calculation flow is summarized in Figure 1a. First, the calculation for the major axis, $c$, will be described. We set the thyroid ROI at the top and bottom of each thyroid lobe on the CT image manually and calculated the centroid coordinates of each ROI: ( $\left.x_{\text {upper, }}, y_{\text {upper }}, z_{\text {upper }}\right)$ and ( $x_{\text {lower, }}, y_{\text {lower, }} z_{\text {lower }}$ ). $c$ was calculated from these coordinates according to Equation 3 . In addition, we obtained the angles, $\varphi$ (formed by $c$ and the coronal plane) and $\rho$ (formed by $c$ and the sagittal plane), as shown in Figure 1b, and Equations 4 and 5.

$$
c[\mathrm{~cm}]=\sqrt{\left(x_{\text {upper }}-x_{\text {lower }}\right)^{2}+\left(y_{\text {upper }}-y_{\text {lower }}\right)^{2}+\left(z_{\text {upper }}-z_{\text {lower }}\right)^{2}}
$$




$$
\varphi[\text { deg. }]=\arctan \left(\frac{y_{\text {upper }}-y_{\text {lower }}}{z_{\text {upper }}-z_{\text {lower }}}\right)
$$

$$
\rho[\text { deg. }]=\arctan \left(\frac{x_{\text {upper }}-x_{\text {lower }}}{z_{\text {upper }}-z_{\text {lower }}}\right)
$$

Subsequently, to calculate $a$ and $b$, we created the cross-sectional plane at the midpoint of the major axis, $c$. Further, according to the definition of the ellipsoid, the cross-sectional plane is perpendicular to the two planes (containing $c$ ). In this study, we limited the plane for defining $a$ and $b$ to the cross-sectional plane at the midpoint of the major axis. Hereinafter, the cross-sectional plane refers to the cross-sectional plane at the midpoint of the major axis. The thyroid ROI of the thyroid region was set manually for this cross-sectional plane (Fig. 1-3). As shown in Figure 2, the centroid of the thyroid ROI in the cross-sectional plane was determined for each thyroid lobe. Furthermore, the combination of the diameter, $a_{\theta}$, and its orthogonal diameter, $b_{\theta}$, at angle $\theta$ were set to pass through the centroid of the thyroid ROI. The diameter of the thyroid in the cross-sectional plane can be obtained innumerable, regardless of whether it passes through the centroid of thyroid ROI. However, in this study, we restricted the diameter to those that pass through the centroid to define a strict ellipsoid. If $\theta$ is rotated from $0^{\circ}$ to $90^{\circ}$, a combination of diameters $\left(a_{\theta}, b_{\theta}\right)$ can be obtained. Thus, a combination of diameters was automatically obtained every $1^{\circ}$ in each thyroid lobe (i.e. 90 combinations). Finally, a total of 8100 thyroid volumes per patient were acquired using 90 combinations of each thyroid lobe as shown in Equation 6.

$$
V_{\text {ellipsoid }}\left(\theta_{L}, \theta_{R}\right)\left[\mathrm{cm}^{3}\right]=\left(\frac{\pi}{6} \times a_{\theta_{L}} \times b_{\theta_{L}} \times c_{L}\right)_{\text {Leftlobe }}+\left(\frac{\pi}{6} \times a_{\theta_{R}} \times b_{\theta_{R}} \times c_{R}\right)_{\text {Rightlobe }}
$$

As a different concept from the one above, Heywood [12] reported that the ellipsoid approximation method can be satisfactorily performed using an equivalent circle diameter (Heywood diameter) as the diameter of the cross-sectional plane. In this study, we also verified 
the accuracy of the approximate volume derived from the equivalent circle diameter (Fig. 3).

197

198

199

200

201

202

203

204

205

206

207

208

209

210

211

212

213

214

215

216

217

218

219

220

221

Assuming that the area of the thyroid ROI in the cross-sectional plane is $S\left(\mathrm{~cm}^{2}\right)$, the equivalent circle diameter, $R(\mathrm{~cm})$, can be expressed as follows using $S$ :

$$
R[\mathbf{c m}]=2 \sqrt{\frac{S}{\pi}}
$$

Equations 8 and 9 is obtained by substituting $R$ into $a$ and $b$ in Equation 6 .

$$
V_{\text {ellipsoid,Heywood }}\left[\mathrm{cm}^{3}\right]=\left(\frac{\pi}{6} \times R_{L} \times R_{L} \times c_{L}\right)_{\text {Leftlobe }}+\left(\frac{\pi}{6} \times R_{R} \times R_{R} \times c_{R}\right)_{\text {Rightlobe }}
$$

$$
V_{\text {ellipsoid,Heywood }}\left[\mathrm{cm}^{3}\right]=\left(\frac{2}{3} \times S_{L} \times c_{L}\right)_{\text {Leftlobe }}+\left(\frac{2}{3} \times S_{R} \times c_{R}\right)_{\text {Rightlobe }}
$$
.

Among the thyroid volumes, $V_{\text {ellipsoid }}\left(\theta_{\mathrm{L}}, \theta_{\mathrm{R}}\right)$, obtainable by the methods described in Figures 2-3 and Equations 6-9, five approximate volumes were compared with those determined three-dimensionally from CT images. These volumes are as follows: (1) the mean volume of the 8100 thyroid volumes, ( $\left.V_{\text {ellipsoid,mean }}\right)$, (2) the volume using the maximum

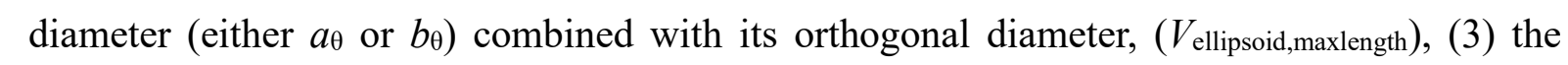

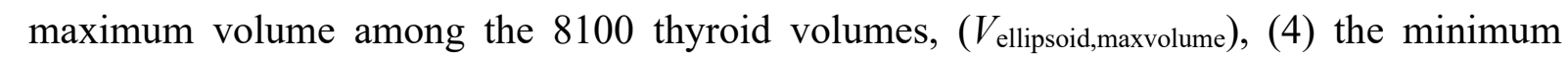

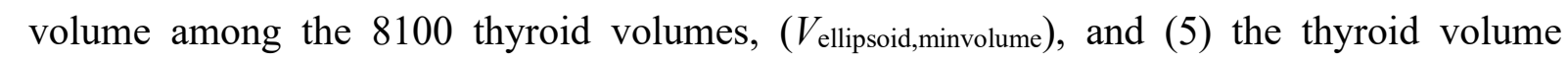

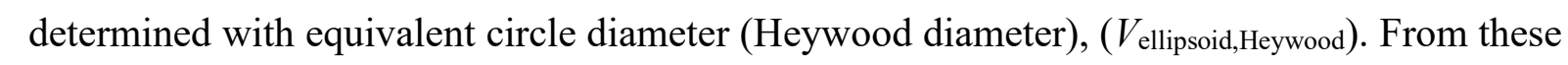
relationships, the accuracy and validity of the ellipsoid approximation method were examined, and the optimal method was identified.

\section{$\underline{\text { Statistical analysis }}$}

We performed a simple regression analysis between the five approximate volumes and the three-dimensionally extracted thyroid volume, i.e., $V_{\mathrm{CT}}$, in the statistics software package, $\mathrm{R}$ 
(version 3.5.1 for Windows). The error rate between the five approximate volumes ( $\left.V_{\text {ellipsoid }}\right)$ and $V_{\mathrm{CT}}$ was obtained using Equation 10.

224

$$
E_{\text {ellipsoid }}[\%]=100 \times \frac{V_{\text {ellipsoid }}-V_{\text {CT }}}{V_{\text {CT }}}
$$

226

227 Furthermore, we calculated a Lin's concordance correlation coefficient (CCC) according to Equation 11 to examine the consistency between $V_{\mathrm{CT}}$ and $V_{\text {ellipsoid }}$ in each patient [13].

229

230

$$
\operatorname{CCC}=\frac{2 \cdot \operatorname{cov}\left(V_{\mathrm{CT}}, V_{\text {ellipsoid }}\right)}{\sigma_{V_{\mathrm{CT}}}^{2}+\sigma_{V_{\text {ellipsoid }}}^{2}+\left(\mu_{V_{\mathrm{CT}}}-\mu_{V_{\text {ellipsoid }}}\right)^{2}}
$$

231

232

where $\operatorname{COV}\left(V_{\mathrm{CT}}, V_{\text {ellipsoid }}\right)$ is the covariance between $V_{\mathrm{CT}}$ and $V_{\text {ellipsoid }}, \sigma_{V_{\mathrm{CT}}}^{2}$ and $\sigma_{V_{\text {ellipsoid }}}^{2}$ are the variances of $V_{\mathrm{CT}}$ and $V_{\text {ellipsoid, }}$ respectively, and $\mu_{V_{\mathrm{CT}}}$ and $\mu_{V_{\text {ellipsoid }}}$ are the mean of $V_{\mathrm{CT}}$ and $V_{\text {ellipsoid, }}$ respectively.

McBride [14] suggested the following scale to describe the correlation based on values of CCC; < 0.90: Poor, 0.90-0.95: Moderate, 0.95-0.99: Substantial, > 0.99: Almost perfect. Similar to the study of Lee et al. [10], we compared the CCC values for the five approximate volumes with McBride's scale. 
Table 1 shows the length of the major axis, $c$, and the angles, $\varphi$ and $\rho$, in each thyroid lobe. The value of $c$ on both thyroid lobes is approximately $6 \mathrm{~cm}$ for all 50 cases. The angle, $\varphi$, is $8.30 \pm$ $3.28^{\circ}$ in the left lobe and $-7.65 \pm 3.14^{\circ}$ in the right lobe. Similarly, the angle, $\rho$, is $0.19 \pm 4.95^{\circ}$ in the left lobe and $1.02 \pm 5.41^{\circ}$ in the right lobe. Figure 4 shows the relationship between $V_{\mathrm{CT}}$ and the 8100 thyroid volumes per patient obtained by systematically combining $a$ and $b$ of each thyroid lobe $\left[V_{\text {ellipsoid }}\left(\theta_{\mathrm{L}}, \theta_{\mathrm{R}}\right)\right]$. The upper end of the error bar represents the maximum thyroid volume ( $V$ ellipsoid,maxvolume), and the lower end represents the minimum thyroid volume ( $V$ ellipsoid,minvolume). Thyroid volumes obtained by the ellipsoid approximation method varied by changing the combination of $a$ and $b$. In particular, the variation in the approximated volume tended to increase as the thyroid volume increased (shaded area in Figure 4).

Table 2 shows the thyroid volumes obtained by three-dimensional volumetry $\left(V_{\mathrm{CT}}\right)$

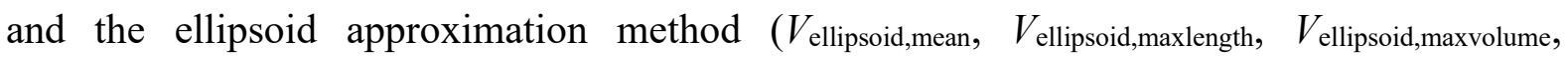

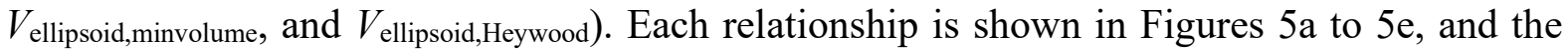
error rates from $V_{\mathrm{CT}}$ and the ellipsoid approximation method ( $E_{\text {ellipsoid,mean, }} E_{\text {ellipsoid,maxlength, }}$ $\left.E_{\text {ellipsoid,maxvolume, }} E_{\text {ellipsoid,minvolume, and }} E_{\text {ellipsoid,Heywood }}\right)$ are shown in Figures 6a to 6e. $V_{\mathrm{CT}}$ as the reference volume was widely distributed between 12.0 to $77.4 \mathrm{~cm}^{3}$. Table 3 lists the values of the correlation coefficient, $\mathrm{CCC}$, and McBride's scale for the thyroid volumes calculated by the ellipsoid approximation method when compared to $V_{\mathrm{CT}}$. All correlations are strong with correlation coefficients of 0.900 or higher. The mean error rate was the highest between $V_{\text {ellipsoid,minvolume }}$ and $V_{\mathrm{CT}}(-23.5 \%)$, and the lowest between $V_{\text {ellipsoid,Heywood }}$ and $V_{\mathrm{CT}}(2.5 \%)$. CCC was used to assess the consistency of each relationship; the highest was for $V_{\text {ellipsoid,Heywood }}$ $\left(0.947\right.$; moderate) and the lowest was for $V_{\text {ellipsoid,minvolume }}(0.672$; poor). 
Discussion

278 Thyroid volumes obtained by the ellipsoid approximation method varied by changing the combination of $a$ and $b$ as shown in Figure 4. In particular, the variation in the approximated volumes tended to increase as the thyroid volume increased. Generally, when performing thyroid volumetry on an ultrasound image, the length of the cross-sectional plane is subjectively determined by the operator. Thyroid volumetry using the ellipsoid approximation method that requires the lengths, $a$ and $b$, is prone to error. According to Figures 5 and 6 , and Table 3, although there was a correlation between $V_{\mathrm{CT}}$ and the ellipsoid approximation volumes, CCC differed depending on the approximate volume (0.672-0.947). $V_{\text {ellipsoid,maxlength }}$

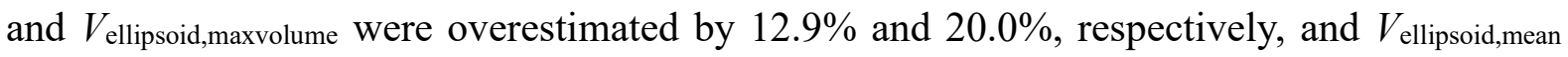

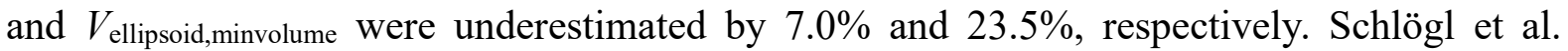
reported that the approximate volume was $11 \%$ larger than the actual volume. Our study supported their findings because they used the maximum diameter to approximate the ellipsoid. In radioiodine therapy for Graves' disease, any error in the measurement of the thyroid volume directly affects the absorbed dose by the thyroid, and thereby the therapeutic effect, as shown in Equation 1. The success rate of internal radioiodine therapy in Graves' disease has been reported by Peters et al. [15] to depend on the thyroid absorbed dose and thyroid weight. By applying these errors to Marinelli's formula (Eq. 1) and calculating backwards with the administered radioactivity, $A$, as a constant, the thyroid absorbed doses, $D$, are reduced by $11.4 \%$ and $16.6 \%$ when $V_{\text {ellipsoid,maxlength }}$ and $V_{\text {ellipsoid,maxvolume are used as thyroid volumes, }}$ respectively. Similarly, when $V_{\text {ellipsoid,mean }}$ and $V_{\text {ellipsoid,minvolume }}$ are used, the thyroid absorbed doses are $7.6 \%$ and $30.8 \%$ in excess of the reference.

$V_{\text {ellipsoid,mean }}$ can be obtained by automatically measuring the volume of the thyroid using a combination of $a$ and $b$ and then averaging these multiple volumes (8100 volumes per patient in this study); thus, the mean error with $V_{\mathrm{CT}}$ could be reduced to $7.0 \%$ because of error

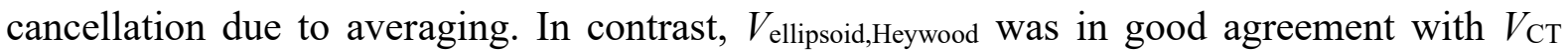
according to the results of single regression analyses and the resultant $\mathrm{CCC}$ values; the mean error with $V_{\mathrm{CT}}$ was $2.5 \%$. There are no reports of past studies in which the thyroid volumes 
were calculated by the ellipsoid approximation method using the equivalent circle diameter.

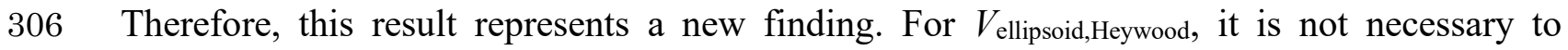
consider the variation due to $a$ and $b$ because this approximated thyroid volume is calculated from the equivalent circle diameter derived from the thyroid ROI on the cross-sectional plane. Therefore, if the ROI can be set accurately in the thyroid region, the accuracy and robustness of the ellipsoid approximation method should be higher than that for the conventional ellipsoid diameters.

We also considered the length of the major axis, $c$. The angle between $c$ and the coronal plane was approximately $8^{\circ}$, and the angle between $c$ and the sagittal plane was approximately $1^{\circ}$, for all cases. At these angles, the error between the projected length of $c$ on the coronal or sagittal plane and the actual length of $c$ was approximately $1 \%$. Lee et. al. measured the length of the major axis on the coronal or sagittal planes and calculated the thyroid volume by the ellipsoid approximation method [10]. Our study supported the validity of measuring the axial length from the coronal or sagittal planes described in their method. The length of the major axis could be measured accurately and easily if we use CT images. An arbitrary cross-sectional image can be obtained using ultrasonography, although this route is prone to subjectivity in measurement. In recent years, it has become possible to acquire volume images with less distortion using three-dimensional ultrasound images. If the knowledge of this study is applied to ultrasound images, thyroid volumetry could be acquired more easily and accurately in the 324 future.

For each volume determined in this study, the error due to the ellipsoid approximation method was in the range, $0-50 \%$, and in some cases the error exceeded $50 \%$. Although it is possible to easily obtain an approximate thyroid volume by the ellipsoid approximation method, the limitation is the approximation itself. Even $V_{\text {ellipsoid,mean }}$ and $V_{\text {ellipsoid,Heywood contains }}$ non-negligible errors due to the approximation. Therefore, we conclude that thyroid volumetry using the ellipsoid approximation cannot be a viable alternative to three-dimensional thyroid volumetry in radioiodine therapy; it is necessary to perform three-dimensional thyroid volumetry using CT or ultrasound images for therapeutic efficacy. 
In this study, we set the cross-sectional plane at the midpoint of the major axis according to the definition of the ellipsoid, and obtained the cross-sectional diameters, $a$ and $b$, on this plane. In clinical practice, the cross-sectional diameter of the thyroid is often obtained from the trans-axial plane of the body (cranial to caudal axis) using CT or ultrasonography. That is, the cross-sectional plane at the midpoint of the major axis in the ellipsoid is inclined with the trans-axial plane of a body (Table 1). In the 50 cases of this study, even if the transaxial plane of a body at the midpoint of the major axis was used in place of the cross-sectional plane at the midpoint of the major axis, the difference between the cross-sectional areas of the two planes was $1 \%$ or less. Therefore, the inclination of the cross-sectional plane cannot be a large variation factor. However, it can be concluded that the cross-sectional diameters $(a, b)$ or area $(S)$ are the main parameters affecting the ellipsoid volume.

Patients with Graves' disease tend to have low CT values in the thyroid. There were some cases where it was difficult to extract thyroid regions in this study. If the CT value of the thyroid is low, the accuracy of the extraction becomes a problem. Although Shu et al. and Lee et al. reported good agreement for the results of three-dimensional thyroid volumetry using contrast-enhanced CT images with the actual thyroid volume [9-10], one of the limitations of our study is that the measurement accuracy of three-dimensional volumetry was not examined. Contrast-enhanced CT scans will improve thyroid visibility and segmentation, but they will delay radioiodine therapy for weeks or months because the contrast media contains iodine [34]. In addition, we need to consider the inter- and intra-operator error of the manually extractedthyroid ROI. In contrast, visibility of the thyroid is improved in ultrasound images. Therefore, it would be easier to extract the region, although determining the cross-sectional plane of the thyroid lobe could be difficult. In our hospital, we measure thyroid volumes using CT images. Therefore, ultrasound images were not available as part of a retrospective study. Further studies are needed to verify the accuracy of our five ellipsoid-approximation-method volumes using ultrasound images to extract reference volume data. 
362 We determined the variation in the approximated thyroid volumes determined by the ellipsoid 363 approximation method using CT images; thyroid volumes varied depending on the diameter or 364 area of the cross-sectional plane. Furthermore, we found that the ellipsoid approximation 365 method using the equivalent circle diameter can determine thyroid volumes in good agreement 366 with those extracted manually by three-dimensional volumetry. However, thyroid volumetry 367 by the ellipsoid approximation method included an error in the range $0-50 \%$ and in some cases 368 the error exceeded 50\%. Consequently, we recommend three-dimensional thyroid volumetry if 369 measurement accuracy is required.

370 
Abbreviations

390 ROI: Region of interest, CCC: Concordance correlation coefficient

391 Declarations

392 Ethics approval and consent to participate:

393 All procedures performed in studies involving human participants were in accordance with the 394 ethical standards of the Ethical Review Committee of Nagoya University Hospital and with the 3951964 Helsinki Declaration and its later amendments or comparable ethical standards. This

396 study was approved by the Ethical Review Committee of Nagoya University Hospital 397 (authorization no. 2018-0179). Since this study analysed clinical data retrospectively, we did 398 not obtain informed consent directly from each patient. Instead of direct informed consent, we 399 published the research contents on the university web page and provided an opt-out 400 opportunity.

\section{Consent for publication}

402 Not applicable.

\section{Availability of data and material:}

404 The datasets used and/or analysed during the current study are available from the 405 corresponding author on reasonable request.

\section{Competing interests:}

407 The authors declare that they have no competing interests.

\section{Funding}

409 Not applicable.

\section{Authors' contributions:}

$411 \mathrm{KK}$ is the guarantor of integrity of the entire study. NF and KK contributed to the study concepts and design. NF and KK contributed to the literature research. NF, KK, SA, and SN

413 contributed to the clinical studies. NF and KK contributed to the experimental studies/data analysis. NF contributed to the statistical analysis. KK and NF contributed to the manuscript preparation. KK contributed to the manuscript editing. All authors read and approved the final manuscript. 


\section{Acknowledgements}

418 The authors thank all current and former Nagoya University Hospital Nuclear Medicine 419 Department staffs who contributed to the acquisition of data included in this study.

420

421

422

423

424

425

426

427

428

429

430

431

432

433

434

435

436

437

438

439

440

441

442

443

444 
1. Smith TJ, Hegedüs L. Graves' Disease. N Engl J Med. 2016; 375:1552-65.

2. Marinelli LD, Quimby EH, Hine GJ. Dosage determination with radioactive isotopes; practical considerations in therapy and protection. Am J Roentgenol Radium Ther. 1948; 59: $260-81$.

3. Stokkel MP, Handkiewicz Junak D, Lassmann M, Dietlein M, Luster M. EANM procedure guidelines for therapy of benign thyroid disease. Eur J Nucl Med Mol Imaging. 2010; 37: $2218-28$

4. Hänscheid H, Canzi C, Eschner W, Flux G, Luster M, Strigari L, Lassmann M. EANM Dosimetry Committee series on standard operational procedures for pre-therapeutic dosimetry II. Dosimetry prior to radioiodine therapy of benign thyroid diseases. Eur J Nucl Med Mol Imaging. 2013; 40: 1126-34

5. Goodwin WE, Cassen B, Bauer FK. Thyroid gland weight determination from thyroid scintigrams with postmortem verification. Radiology. 1953; 61: 88-92.

6. Allen HC Jr, Goodwin WE. The scintillation counter as an instrument for in vivo determination of thyroid weight. Radiology. 1952; 58: 68-79.

7. Malago R, D'Onofrio M, Ferdeghini M, Mantovani W, Colato C, Brazzarola P, Motton M, Mucelli RP. Thyroid volumetric quantification: comparative evaluation between conventional and volumetric ultrasonography. J Ultrasound Med. 2008; 27:1727-33.

8. Hermans R, Bouillon R, Laga K, Delaere PR, Foer BD, Marchal G, Baert AL. Estimation of thyroid gland volume by spiral computed tomography. Eur Radiol. 1997; 7: 214-6.

9. Shu J, Zhao J, Guo D, Luo Y, Zhong W, Xie W. Accuracy and reliability of thyroid volumetry using spiral CT and thyroid volume in a healthy, non-iodine-deficient Chinese adult population. Eur J Radiol. $2011 ; 77: 274-80$.

10. Lee SJ, Chong S, Kang KH, Hur J, Hong BW, Kim HJ, Kim SJ. Semiautomated thyroid volumetry using 3D CT: prospective comparison with measurements obtained using $2 \mathrm{D}$ ultrasound, 2D CT, and water displacement method of specimen. AJR Am J Roentgenol. 2014; 203: W525-32. 
11. Schlögl S, Werner E, Lassmann M, Terekhova J, Muffert S, Seybold S, Reiners C. The use of three-dimensional ultrasound for thyroid volumetry. Thyroid. 2001; 11: 569-74.

12. Heywood H. Numerical definitions of particle size and shape. Chem. Ind. 1937; 56: 14954

13. Lin LI. A concordance correlation coefficient to evaluate reproducibility. Biometrics. 1989; 45: $255-68$.

14. McBride GB. A proposal for strength-of-agreement criteria for Lins Concordance Correlation Coefficient. NIWA Client Report 2005: HAM2005-062.

15. Peters H, Fischer C, Bogner U, Reiners C, Schleusener H. Radioiodine therapy of Graves' hyperthyroidism: standard vs. calculated ${ }^{131}$ iodine activity. Results from a prospective, randomized, multicentre study. Eur J Clin Invest. 1995; 25: 186-93

.

5

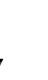

8

9

.

.

3

4

5


502 Table 1. Length of the major axis, $c$, and the angles, $\varphi$ and $\rho$, in each patient's thyroid lobe.

503 Table 2. Thyroid volumes calculated by three-dimensional volumetry $\left(V_{\mathrm{CT}}\right)$ and the ellipsoid approximation method ( $V_{\text {ellipsoid,mean, }} V_{\text {ellipsoid,maxlength, }} V_{\text {ellipsoid,maxvolume}}, V_{\text {ellipsoid,minvolume, and }}$

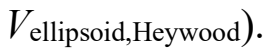

Table 3. Correlation coefficient, $\mathrm{CCC}$, and McBride's scale between $V_{\mathrm{CT}}$ and each approximate volume. CCC for assessing the consistency of each relationship was above 0.900 for $V_{\text {ellipsoid,mean }}$ and $V_{\text {ellipsoid,Heywood. }}$

Fig. 1. a) Calculation method for thyroid volumes by the ellipsoid approximation method. Calculation method for the length of the major axis, $c$, and the angles, $\varphi$, and $\rho$, in each patient's 511 thyroid lobe.

512 Fig. 2. Measurement of arbitrary diameters and their corresponding orthogonal diameters ( $a$ and $b$ ) on the cross-sectional plane at the midpoint of the major axis. Four approximate volumes

514 ( $V_{\text {ellipsoid,mean, }} V_{\text {ellipsoid,maxlength, }} V_{\text {ellipsoid,maxvolume, and }} V_{\text {ellipsoid,minvolume}}$ ) were obtained from $a$ and $515 \quad b$.

516 Fig. 3. Ellipsoid approximation method for thyroid volumes using the equivalent circle 517 diameter (Heywood diameter).

518 Fig. 4. Relationship between $V_{\text {Cт }}$ and the 8100 thyroid volumes obtained by combining $a$ and $519 b$ of each thyroid lobe $\left[V_{\text {ellipsoid }}\left(\theta_{\mathrm{L}}, \theta_{\mathrm{R}}\right)\right]$. The upper and lower end of the error bar represents the maximum and the minimum thyroid volume, respectively. The shaded area indicates an area surrounded by the regression lines of $V_{\text {ellipsoid,maxvolume }}$ and $V_{\text {ellipsoid,minvolume }}$ (Corresponding to Fig. $5 \mathrm{c}$ and $5 \mathrm{~d}$, respectively). The variation in the approximate volumes tended to increase as the thyroid volume increased.

Fig. 5. Relationship between $V_{\mathrm{CT}}$ and the five approximate volumes ( $V_{\text {ellipsoid,mean, }}$

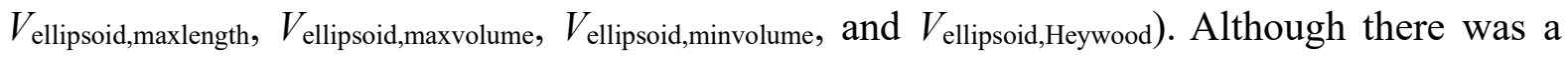
strong correlation between $V_{\mathrm{CT}}$ and these approximate volumes, the slopes of the regression equation were different for each relationship (0.79-1.20). For this reason, $\mathrm{CCC}$ differed for each approximate volume (0.672-0.947). 
529 Fig. 6. Error rate between $V_{\mathrm{CT}}$ and the five approximate volumes $\left(E_{\text {ellipsoid,mean, }} E_{\text {ellipsoid,maxlength, }}\right.$

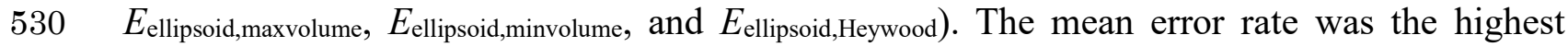

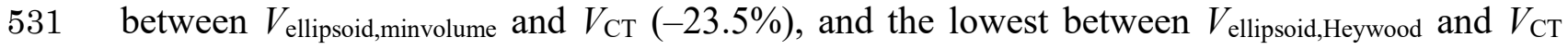
$532(2.5 \%)$ 
Figures

a)

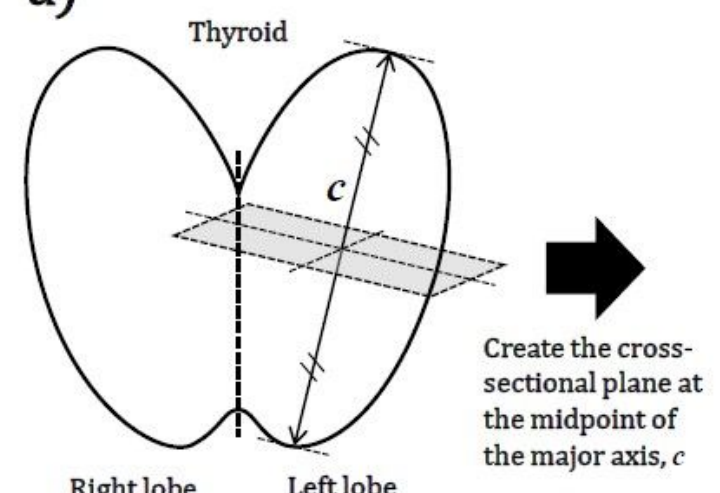

Right lobe

Left lobe
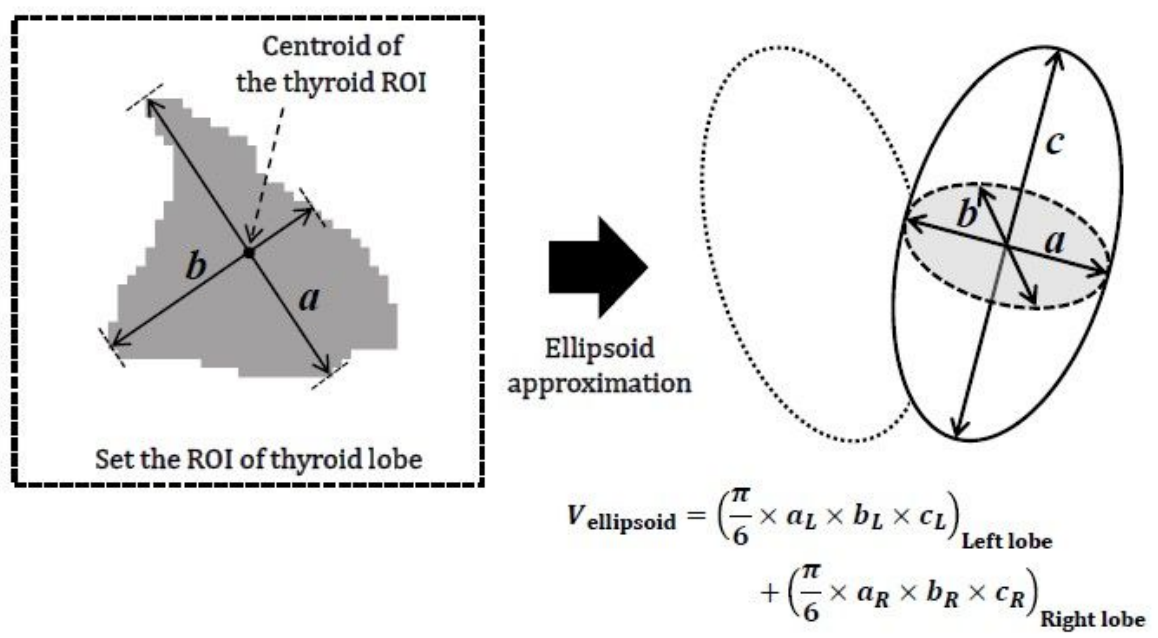

b)

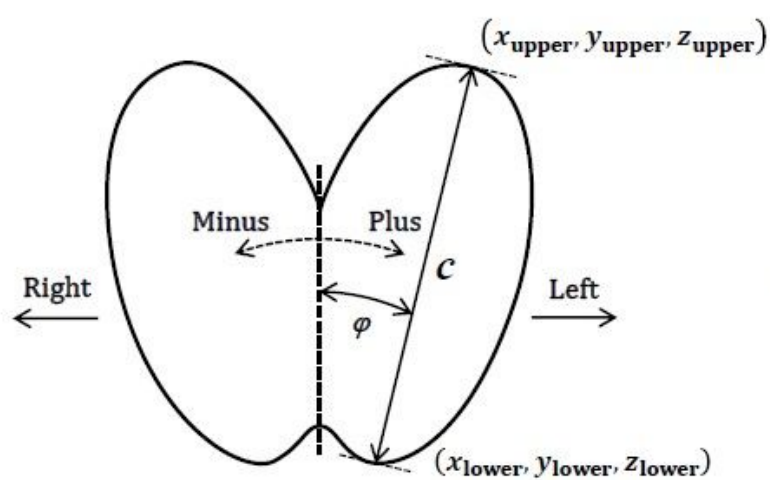

Coronal plane

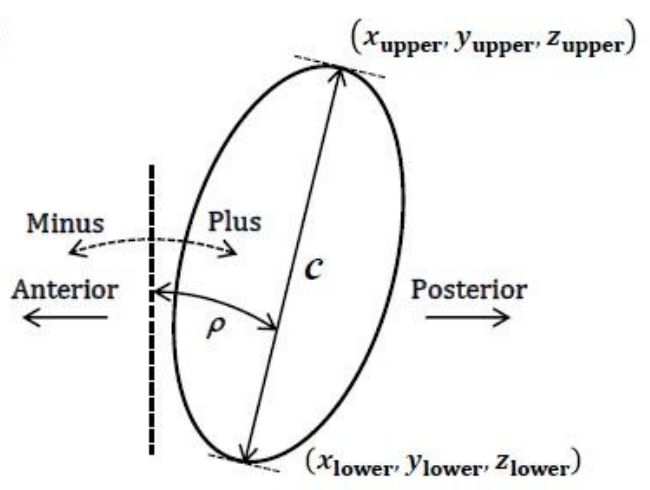

Sagittal plane

\section{Figure 1}

a) Calculation method for thyroid volumes by the ellipsoid approximation method. b) Calculation method for the length of the major axis, $c$, and the angles, $\varphi$, and $\rho$, in each patient's thyroid lobe. 


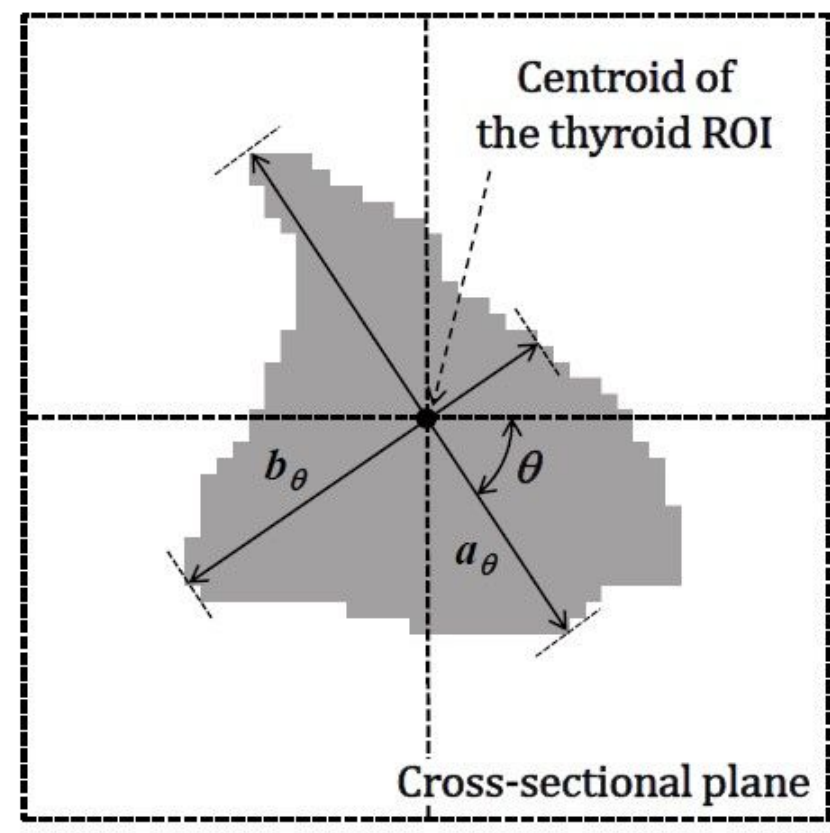

\section{Ellipsoid approximation}

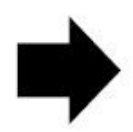

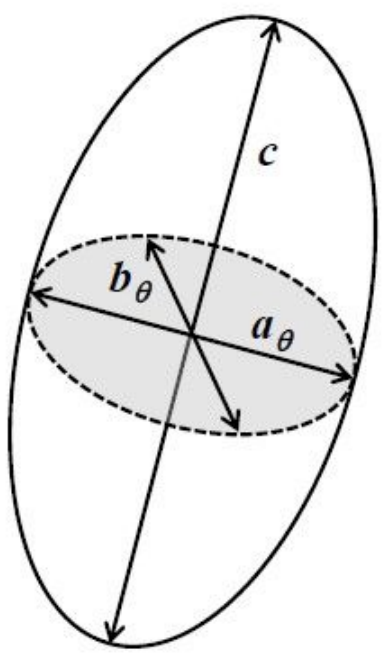

$$
V_{\text {ellipsoid }}\left(\theta_{L}, \theta_{R}\right)=\left(\frac{\pi}{6} \times a_{\theta_{L}} \times b_{\theta_{L}} \times c_{L}\right)_{\text {Left lobe }}+\left(\frac{\pi}{6} \times a_{\theta_{R}} \times b_{\theta_{R}} \times c_{R}\right)_{\text {Right lobe }}
$$
(8100 volumes)

$$
\text { (90 volumes) }
$$

$$
\text { (90 volumes) }
$$

The Mean volume of the 8100 thyroid volumes

The volume using the maximum diameter (either $a_{\theta}$ or $b_{\theta}$ ) combined with its orthogonal diameter The maximum volume among the 8100 thyroid volumes The minimum volume among the 8100 thyroid volumes

: $V_{\text {ellipsoid,mean }}$

: $V_{\text {ellipsoid,maxlength }}$ : $V_{\text {ellipsoid,maxvolume }}$ $: V_{\text {ellipsoid,minvolume }}$

Figure 2

Measurement of arbitrary diameters and their corresponding orthogonal diameters ( $a$ and $b$ ) on the crosssectional plane at the midpoint of the major axis. Four approximate volumes (Vellipsoid,mean, Vellipsoid,maxlength, Vellipsoid,maxvolume, and Vellipsoid,minvolume) were obtained from $a$ and $b$. 


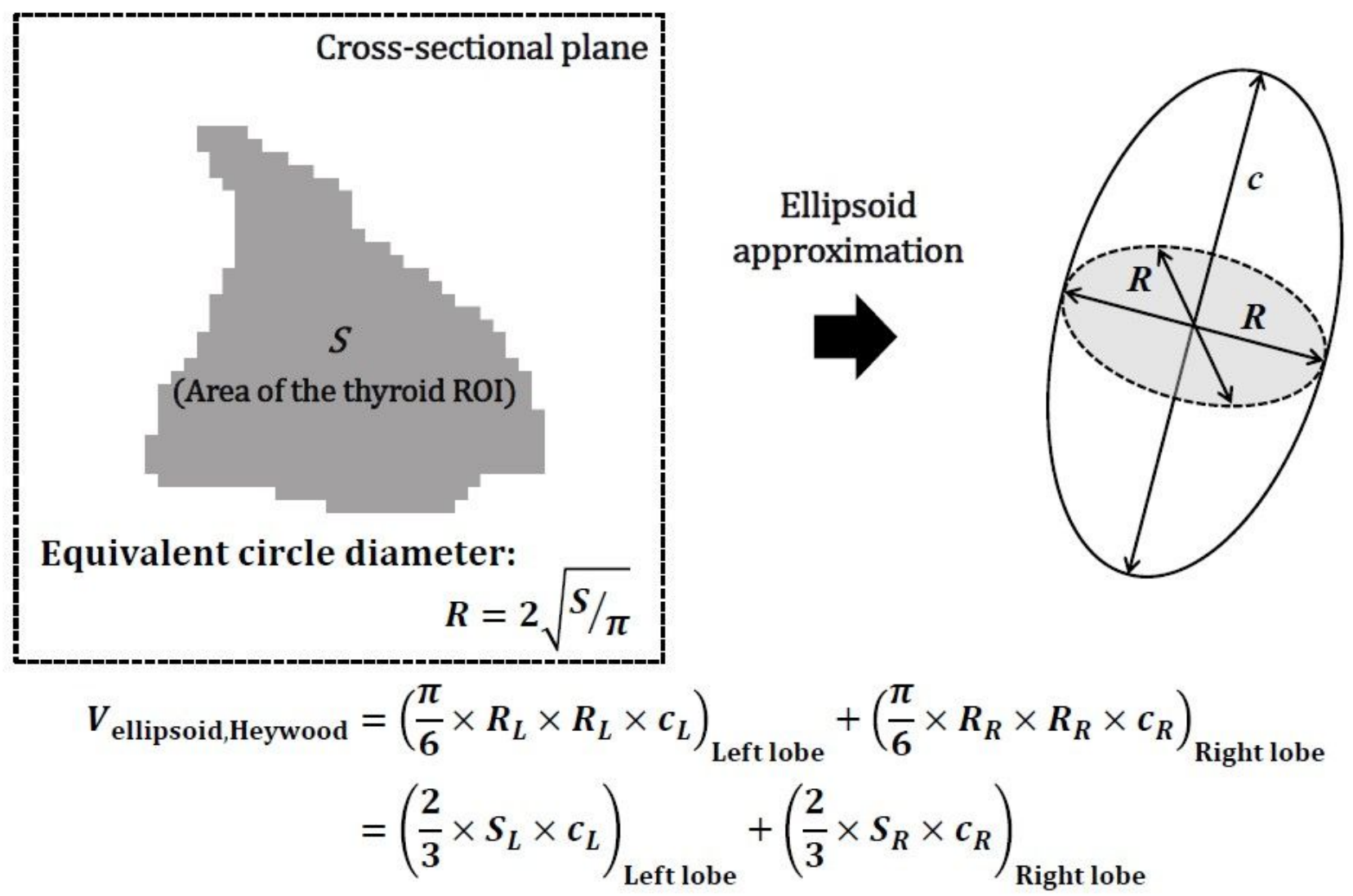

Figure 3

Ellipsoid approximation method for thyroid volumes using the equivalent circle diameter (Heywood diameter). 


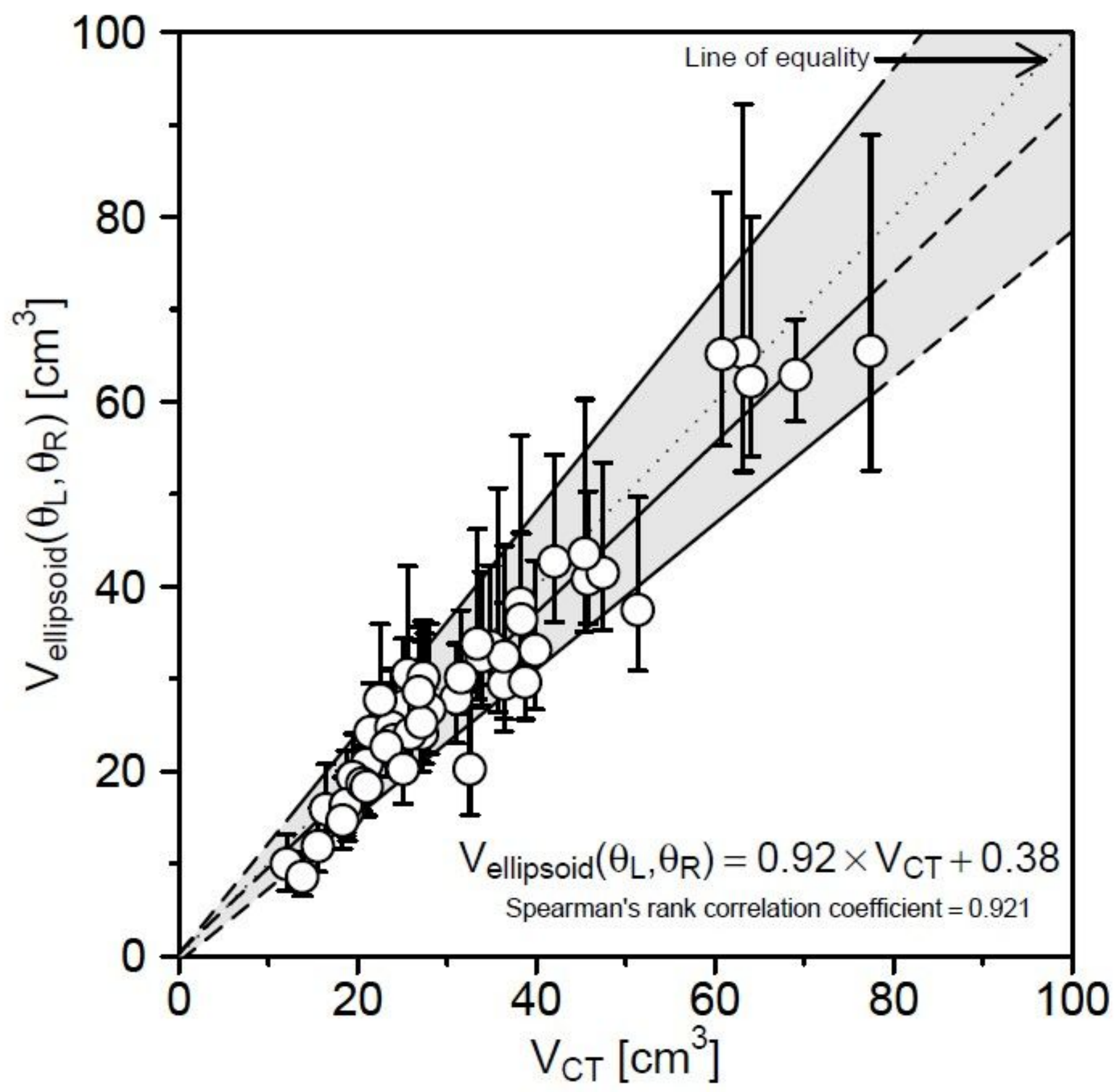

Figure 4

Relationship between VCT and the 8100 thyroid volumes obtained by combining a and $b$ of each thyroid lobe [Vellipsoid $(\theta \mathrm{L}, \theta \mathrm{R})]$. The upper and lower end of the error bar represents the maximum and the minimum thyroid volume, respectively. The shaded area indicates an area surrounded by the regression lines of Vellipsoid,maxvolume and Vellipsoid,minvolume (Corresponding to Fig. 5c and 5d, respectively). The variation in the approximate volumes tended to increase as the thyroid volume increased. 

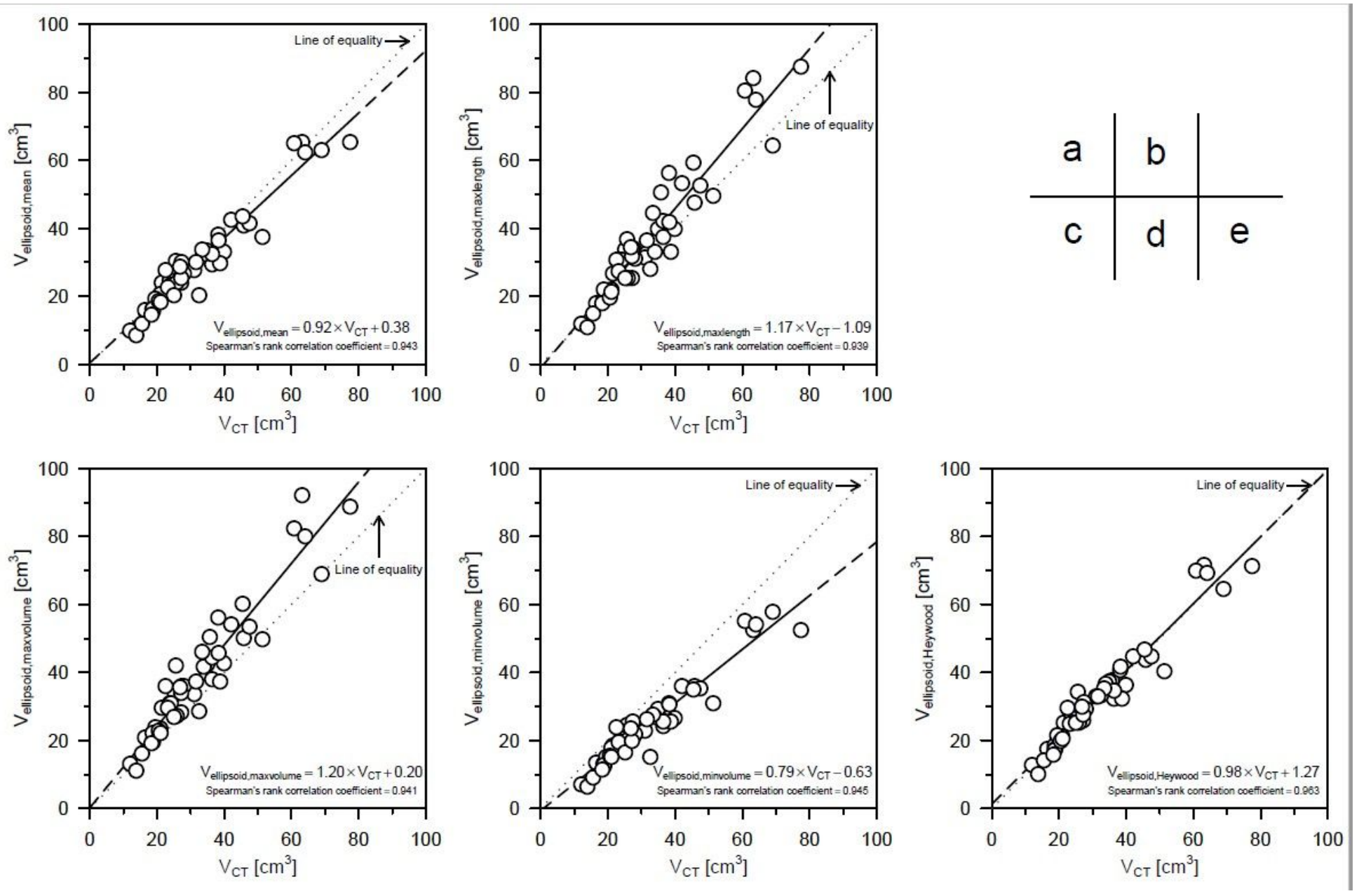

Figure 5

Relationship between VCT and the five approximate volumes (Vellipsoid,mean, Vellipsoid,maxlength, Vellipsoid,maxvolume, Vellipsoid,minvolume, and Vellipsoid,Heywood). Although there was a strong correlation between VCT and these approximate volumes, the slopes of the regression equation were different for each relationship $(0.79-1.20)$. For this reason, CCC differed for each approximate volume (0.672-0.947). 

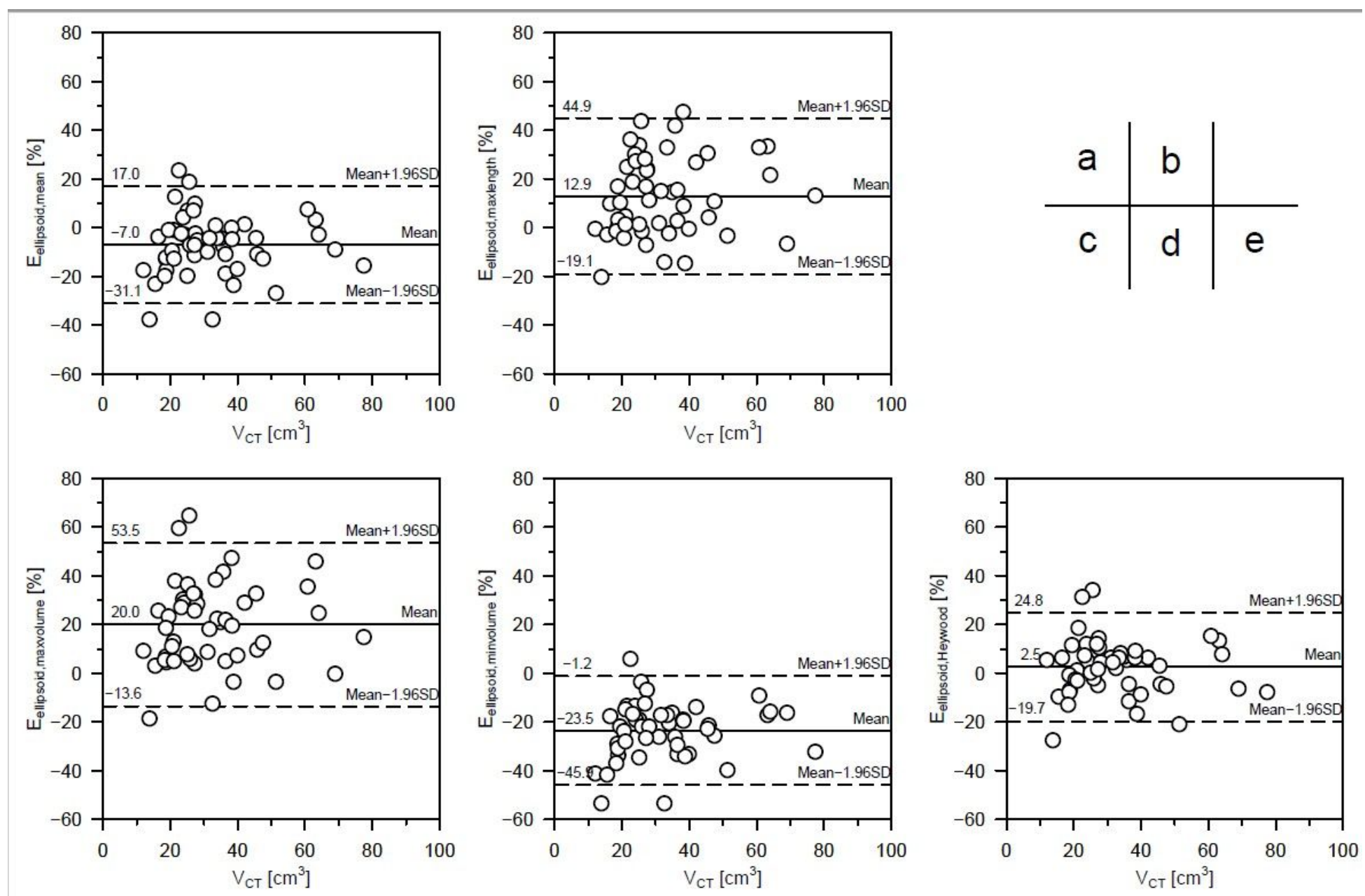

Figure 6

Error rate between VCT and the five approximate volumes (Eellipsoid,mean, Eellipsoid,maxlength, Eellipsoid,maxvolume, Eellipsoid,minvolume, and Eellipsoid,Heywood). The mean error rate was the highest between Vellipsoid,minvolume and VCT (-23.5\%), and the lowest between Vellipsoid,Heywood and VCT $(2.5 \%)$. 